\title{
Being there when it happens: A novel approach to sampling reflectively observed experience
}

\author{
Urban Kordeš, Ema Demšar \\ University of Ljubljana, Center for Cognitive Science, Slovenia
}

\section{A R T I C L E I N F O}

\section{Keywords:}

Empirical phenomenology

Experience sampling

Meditation-based research

Reflective awareness

Contemplative phenomenology

Qualitative research

\begin{abstract}
A B S T R A C T
Examining a number of recently developed methods for acquiring first-person data on consciousness, we detect a lack of sensitivity for distinguishing the experiential moments in which the experiencing person was reflectively attending to her ongoing experience. In order to address this gap, we introduce a novel research format for obtaining data on lived experience, combining random sampling of experience with a subsequent retrospective examination of acquired samples in the form of dialogical phenomenological inquiry. The proposed approach aims at the examination of reflectively observed experiential moments and is based on researchers' iterative cultivation of the phenomenological attitude. Drawing upon results from a longitudinal study of the potential of meditation as a tool for examining consciousness, we address the epistemological and methodological challenges of the proposed approach, discuss its applicability and research potential, as well as examine the characteristics and validity of phenomenal data thus acquired.
\end{abstract}

“... every good student of cognitive science who is also interested in issues at the level of mental experience, must inescapably attain a level of mastery in phenomenological examination in order to work seriously with first-person accounts." (Varela, 1996, pp. 346-347)

In his proposal for a neurophenomenological research program, Francisco Varela (1996) famously called for developing a community of researchers committed to a sustained and disciplined cultivation of the skill of phenomenological examination. While often cited, this suggestion has rarely been actualized in the context of research in psychology and cognitive science. One reason for this might be that when attempting to acquire a mastery in phenomenological examination, it is not clear where to turn for instructions: to philosophical phenomenology, contemplative literature, or perhaps to one among more recently developed first-person approaches in cognitive science? In order for cognitive scientists to master and meaningfully employ the skill of investigating lived experience - especially when the aim is to acquire empirical data that can be used in the scientific context - it appears necessary to first articulate what (empirical) phenomenological examination actually consists in, and how it can be cultivated within a group of consciousness researchers.

In this article, we propose a research format for examining, refining, and employing the process of phenomenological inquiry in the context of empirical research in psychology and cognitive science. The approach, which we call sampling reflectively observed experience (SROE), is aimed at acquiring empirical data on lived experience (i.e., phenomenal data; cf. Varela \& Shear, 1999), and grounded in combining insights and techniques from three lines of inquiry which have, each of them in its own way, long pursued systematic examination of lived experience: contemplative studies, the phenomenological tradition, and empirical first-person methods in psychology and cognitive science. Situated at this intersection, the SROE research format is perhaps best described as belonging to the newly emerging field of contemplative phenomenology (cf. Sparby, 2015).

The methodological framework of SROE is based on two main tenets. The first tenet is a systematic cultivation of attending to ongoing experience with an attitude which, in different traditions, has been described as attentive, reflective, mindful, or phenomenological (see section 3): an attitude by which the experiencing person, rather than being absorbed in the contents of her ongoing experience, maintains an attentive

\footnotetext{
* Corresponding author.

E-mail addresses: urban.kordes@pef.uni-lj.si (U. Kordeš), ema.demsar@pef.uni-lj.si (E. Demšar).
} 
awareness of their mode of appearance (i.e., the structure of experience) at the time of their unfolding. While different approaches to attending to ongoing experience have already been put forward in various meditation traditions, in SROE, the real-time reflective observation ${ }^{1}$ does not rely on any particular meditation technique or otherwise predefined instruction. Instead, the capacity for reflective observing is regarded as a skill that has to be intimately understood, iteratively developed, and critically examined within an intersubjective environment of a group of co-researchers. $^{2}$

The second tenet of SROE's methodology is the acquisition of descriptions of researchers' ongoing experience in selected moments of their pursuit of reflective observation. This is achieved by incorporating two main techniques: (1) sampling the ongoing experience of a group of researchers at random moments during periods in which they are attempting such reflective observation (i.e., during meditation practice, see footnote 2), and (2) interview-based phenomenological examination of the subset of acquired samples in which the researchers in fact report on reflectively attending to their ongoing experience.

SROE research format was developed throughout a year-long exploratory study investigating the potential of meditation practice as an instrument for researching experience. ${ }^{3}$ One of the goals of this study was to examine the kind of practice that would be suitable for researching experience in the context of cognitive science, and to develop the corresponding methodology for acquiring phenomenal data. The aim of the present article is to present SROE as a product of this development: a novel meditation-based methodological framework for the study of lived experience that can be systematically adopted by a group of consciousness researchers.

Compared to other contemporary approaches for researching experience, SROE research format is particularly well-suited for dealing with two fundamental challenges encountered in the construction and validation of any psychological instrument for acquiring phenomenal data.

The first challenge is that producing valid descriptions of experience appears to rely, at least in part, on the experiencing subjects' training in their observational and reporting skills. In their everyday lives, people do not pay much attention to their lived experience, and even when attempting to naively describe it, tend to be systematically mistaken about it (Hurlburt, 2011) and easily led to generate false reports (e.g., Johansson et al., 2005; Loftus et al., 1978; Nisbett \& Wilson, 1977). As has been famously pointed out by Edmund Husserl, we usually relate to the world through the prism of the so-called natural attitude (Husserl, 1983): a web of commonsensical beliefs about the independent existence and structure of the world (including beliefs about experience) that, by focusing on what experience is purportedly about, conceals the way in which it actually appears to us. Relatedly, many researchers throughout the history of the scientific study of experience - most notably Varela (1996), whose quotation set the tone for the present article - have insisted that the skill of observing and accurately describing experience, like any know-how, can only be acquired through systematic training. Aimed at the collaborative examination and cultivation of the observational attitude necessary for investigating

\footnotetext{
${ }^{1}$ In this article, we use the expression observing experience in a broad sense, interchangeably with the expression attending to experience. We use of the term reflective observation to refer to the real-time attentive attending to experience at the moment of its unfolding (See section 3 for a more detailed discussion on and explanations of these terms).

${ }^{2}$ For the sake of brevity, periods in which a group of co-researchers are pursuing the practice of reflectively observing their ongoing experience will be hereon after called meditation practice (see section 4 for a discussion of the relationship between contemplative and phenomenological traditions and empirical first-person study of experience).

3 The methodology and selected results, as well as the ethnographic account of the research process (including the iterative development of the research design leading to SROE methodology) have been published in Kordeš et al. (2019).
}

experience within a group of co-researchers, SROE (to our knowledge) presents one of the first research formats suitable for developing the skill of phenomenological examination in an intersubjective research environment.

The second challenge in developing an instrument for acquiring phenomenal data is that the process of examining experience appears to unavoidably interfere with its target, altering - or even constructing the very phenomenon that it is claiming to examine. The problem, as described by Varela and Jonathan Shear, is the following: "How do you know that by exploring experience with a method you are not, in fact, deforming or even creating what you experience? Experience being what it is, what is the possible meaning of [its] examination?" (Varela \& Shear, 1999, p. 13). Contemporary techniques for acquiring phenomenal data try to deal with this so-called excavation fallacy (Depraz et al., 2003; Varela \& Shear, 1999) in different ways, for instance by instructing participants to report, in real-time, on their cognitive processes while attempting to minimize the interference of their examination (e.g., the "non-reactive verbalization of thoughts" in think-aloud protocols; Ericsson, 2003; cf. Ericsson \& Simon, 1993), or by examining an already transpired experience from the past in a later retrospective interview (e. g., the descriptive experience sampling, Hurlburt, 2011, and the micro-phenomenological approach, Petitmengin, 2006).

SROE research format addresses the problem of excavation in a different way: by recognizing it as an inherent feature of acquiring phenomenal data. Namely, we maintain that in any approach to examining lived experience, the act of observing (and reporting) experience does not merely retrieve information, but constructively co-determines what is being observed and reported (see section 5; Kordeš \& Demšar, 2018). While this constructive dimension of examining experience cannot be circumvented, SROE has the advantage of enabling a detection and articulation of the way in which the examination is carried out. By cultivating real-time reflective awareness of one's experiential field as a whole - including the aspects of experience related to one's observational and attentional activity - SROE aims at describing the attitude with which the experience is being observed as an integral part of the acquired phenomenal data.

By means of integrating the practice of attending to the ongoing experience with a subsequent retrospective examination of this experience in a phenomenological interview, SROE requires the examined experience to be lived through with attentive awareness, and as such presents a methodological invention in the fields of first-person research and contemplative phenomenology. With its requirement for implementing an iterative and longitudinal process of cultivating reflective observation, the proposed research format is likely to require complex and demanding research designs. At the same time, however, its specificity enables an investigation of phenomena that are less available for other existing methods in first-person research. In particular, SROE research format (as we will further explain in the last section) is wellsuited for examining the following types of experience:

\footnotetext{
- Spontaneously emerging experiential phenomena, such as mindwandering;

- Meditation-related experiential processes and states;

- Phenomena typically hidden in the background of experience, such as felt sense (Gendlin, 1996), or existential feelings (Ratcliffe, 2005);

- Invariant (i.e., intersubjectively and intersituationally stable) experiential structures that are often thought underlie the majority of everyday experience.
}

In order to ground the methodological guidelines (and epistemological considerations) for SROE in empirical findings of our already conducted research, this article has an atypical structure. Rather than first laying out the theoretical foundations of the proposed approach, we start by summarizing the relevant elements of the study (partially also reported in Kordeš et al., 2019) whose methodological development and selected findings support SROE research format (section 1), and go on to 
outline the main methodological parameters of SROE (section 2). In sections 3 , 4, and 5 , we provide a theoretical grounding and explanation of the methodological requirements of SROE research format. We clarify the notion of reflective observation of ongoing experience and relate it to the kind of phenomenological examination entailed in SROE (section 3 ); we describe the procedure for the collaborative cultivation of reflective observation in a group of co-researchers (section 4); and we explicate the non-representationalist epistemological framework for understanding the acquired phenomenal data (section 5). In the last two sections, we return to practical guidelines for SROE research format, explaining the notion of co-researchers and the proposed understanding of phenomenological interview as a dialogical investigation (section 6), and finally, discussing the issue of applicability and reliability of SROE, as well as its research potential within the field of first-person approaches to the study of consciousness (section 7).

\section{Study on meditative practice as a tool for investigating consciousness: summary of research design and relevant findings}

The methodological framework we present in this article was developed throughout a longitudinal study aiming to open-endedly investigate the potential of meditation practice as an instrument for researching experience in the context of cognitive science. The study was carried out by a group of seven participants, here called co-researchers or researchers (the choice of these terms is explained below in section 6), who remained constant throughout its year-long course. All participating researchers were familiar with basic ideas of the phenomenological approach as well as with observing and reporting on their own lived experience; they were either trained or training in methods of empirical phenomenology. Throughout the course of the study, all researchers participated in specifying the ongoing focus of research and in carrying out primary qualitative analysis of acquired phenomenal data. In accordance with the ambition to develop an approach that could eventually be employed by researchers in the context of cognitive science, and since we wanted to start our investigation from a theoretically naïve and conceptually unrestricted position, the recruited researchers had different levels of prior experience with meditation: while some were completely new to the practice, others had - at the time of the study - practiced for over a decade.

The study had three related aims: first, to identify and describe the kind of meditative practice that would be suitable for investigating lived experience and could be systematically adopted by a group of consciousness researchers in the context of cognitive science; second, to see whether systematic employment of such meditation-based examination of experience could identify and study phenomena observed across different researchers; and third, to provide an ethnographic account of the research process itself, including the descriptions of the actual experience of researchers engaged in the meditation-based examination of experience.

For results and considerations related to the third aim, see Kordeš et al. (2019), in which we present selected results of the investigation of researchers' experience during meditation practice, as well as detail the employed methodology and its iterative development throughout the course of the study. As one of the aims of the study, this purposeful development of the research format consisted of iteratively refining two related, but separate aspects of the methodological framework: (1) researchers' approach to meditation-based reflective observation of their ongoing experience; (2) the approach to acquiring data on experience during meditation.

While the present article takes up and elaborates on the first aim of the study, this section, in particular, summarizes the research design of the study (Box 1), as well as selected results of the study. The gradual refinement of the research design, reported in more detail in Kordeš et al. (2019), resulted in the development of the SROE research format.

The samples acquired throughout the course of the study showed a variety of different experiences during meditation, ranging from experiences similar to those described in meditation literature (e.g., states of meditative presence, feeling of oneness), to more everyday experiences such as remembering a conversation with one's partner, planning next week's activities, listening to another meditator's loud breathing, or dealing with annoyance with the pain in one's knee. Compare for instance the following two examples, the first reporting a typical state of meditative presence, and the second reporting a typical example of mind-wandering:

\#1 “Completely present. A gestalt feeling of all the experiences together. Everything is infused with the feeling of freshness and enthusiasm." (C1-R5-D5-5)

\#2 'I'm thinking (in inner speech) about the lemonade downstairs. At the same time, I'm seeing an image of the glass of lemonade on the kitchen counter; on the horizon there is already the upcoming feeling of what the lemonade would taste like and also what it would look like." (C3-R4-D1-6), ${ }^{45}$

In addition to revealing a spectrum of different contents of experience, the qualitative analysis of 350 experience samples acquired in the third, fourth, and fifth retreat ${ }^{6}$ showed another important difference across the samples: the way in which the researches were relating to their ongoing experience - whatever its content - at the moment of the sampling. In some of the samples (e.g. \#1), the researchers reported being in continuous contact with their experiential landscape, attentively aware of their ongoing experience. ${ }^{7}$ In other samples (e.g. \#2), the researchers reported their attention being absorbed in the contents of their ongoing experience - whatever their experience, became aware of how it appeared to them only at (or after) the sampling signal. ${ }^{8}$

Preliminary analysis of the samples soon revealed various degrees to which the researcher could be attentively aware of her ongoing experience, as well as various modes of such attentive awareness. Consider the following sample, reporting an instance of mind-wandering that the researcher was attentively aware of already at the moment of its unfolding (and not, as in \#2 above, only after the sampling signal):

\#3 "Focus on the belly, at the same time I have the feeling of inner stability, together with which there appears an image of bricks being assembled into a triangle: the lower part of the triangle is laying on the lower part of my pelvis. I am observing this image and how it is appearing; at the same time, I am already slightly carried towards being absorbed in it. But the focus on the belly remains." (C4-R4-D24)

\footnotetext{
${ }^{4}$ Samples presented in this article are excerpts from the researchers' (usually more extensive) notes about their experience just before the moment of the sampling. All samples originate from the meditation study (Kordeš et al., 2019); each is labeled with an identification code in the form of Cx-Rx-Dx-X, where Cx is the co-researcher's code (for the notion of the co-researcher, see section 6 below), Rx the consecutive number of the retreat at which the experience was sampled, Dx the consecutive day of sampling, and $\mathrm{X}$ the consecutive meditation session (of the day) that the sample was taken in. The samples were written in Slovene; excerpts from them were translated into English with minimal editing and the anonymization of personal information.

${ }^{5}$ Note that although they often use the present tense (and sometimes past tense), all descriptions refer to the researcher's experience from just before the moment of the sampling signal. In \#2, the fact that thinking appeared in inner speech was, for instance, noticed only after the sampling signal, rather than already at the time of its unfolding.

${ }^{6}$ Results of this analysis are presented in Kordeš et al. (2019). Samples acquired in the first and second retreat were excluded from the analysis, as the sampling approach in those two retreats was still being refined and therefore yielded samples not entirely comparable to those acquired later in the study.

7 Coded as Presence in the final results of analysis, these samples encompassed $17 \%$ of the totality of analyzed samples (see Kordeš et al., 2019).

${ }^{8}$ Coded as Mind-wandering in the final results of analysis, these samples encompassed $70 \%$ of the totality of analyzed samples (see Kordeš et al., 2019).
} 


\section{Box 1}

Summary of the methodology employed in Kordeš et al. (2019). For details on the selection and development of different aspects of this research design, see the original article.

\section{Summary of methodology employed in Kordeš et al. (2019)}

Silent meditation retreats. The study was carried out in the form of five silent meditation retreats, ranging in length from one day to seven days. Each day consisted of 8-10 35-min-long group meditation sessions. Meditation was practiced in a sitting position and with eyes closed.

Cultivating observation through collaborative exploration of observational attitude. We experimented with different approaches to observing experience throughout the course of the study: from trying out standard meditation techniques to following instructions for phenomenological observation. In the end, the approach that proved most fruitful was to enable a refinement of each researcher's practice of phenomenological observation through the use of video recordings of phenomenological interviews about the carried out with their co-researchers (details on individuals' approaches to observing experience, explanation of the role of viewing interview recordings, and a further explanation of how - as well as why - the adopted approach differed from employing standard approaches to meditating will be elaborated in section 4 below).

Data acquisition: Sampling experience. Data on ongoing experience was acquired via random sampling modeled after Russell Hurlburt's (2011) descriptive experience sampling method. At a semi-randomly selected moment during each meditation session, all present researchers were prompted by the sound of an electronic gong to note down their experience as it was unfolding just before the sampling sound; after taking notes, they continued meditating until the completion of the full $35 \mathrm{~min}$ of the session.

Data acquisition: Phenomenological interviews. Randomly selected experience samples from each researcher were further explored in phenomenological interviews based on the interviewed researcher's sampling notes. The interviews lasted around 40-90 min and were conducted according to the general guidelines for phenomenological interviews as outlined in Petitmengin (2006), but took a more focused approach of dialogical research (explained below in section 6).

Of the samples that could not be straightforwardly coded as either fully-blown (meditative) presence with experience or complete lack of attending to ongoing experience, ${ }^{9}$ especially interesting for developing the SROE research format are those in which the researcher was experiencing processes and states that are often observed in people's everyday experiential lives - phenomena such as thinking, inner speech, inner hearing, seeing mental imagery, bodily sensations, and other kinds of sensory awareness (compare to "frequently occurring phenomena of experience" in Heavey \& Hurlburt, 2008) - but was attentively aware of their unfolding at the moment of the sampling.

Informed by the variety of ways of relating to one's ongoing experience, we developed the SROE research format to specifically target moments of experience in which the researcher was attentively aware of her ongoing experience in the moment just before the sampling signal. Compared to the research design of Kordeš et al. (2019) described in Box 1 , in SROE, the samples of experience further investigated in a phenomenological interview are not selected randomly, but are limited to those characterized by attentive awareness that enabled the researcher to reflectively observe her ongoing experience at the moment of the sampling. In what follows, we describe the SROE research format and ground it in empirical data acquired in Kordeš et al. (2019).

\section{SROE: summary of the methodological parameters}

SROE is a research format for acquiring phenomenal data, based on sampling the experience of a group of researchers during sessions in which they attempt to observe whatever occurs in their consciousness with reflective awareness. Some of the sampled moments are subsequently further explored in phenomenological interviews. The experience caught in those samples can be described by two defining characteristics:

The experience was being attended to when it happened - as it was being lived through, and not after it had already transpired. (This

\footnotetext{
${ }^{9}$ Coded as In-between in the final results of analysis, these samples encompassed $11 \%$ of the totality of analyzed samples (see Kordeš et al., 2019).
}

distinguishes SROE from retrospective strategies such as microphenomenology or descriptive experience sampling.)

The experience was being attended to with the phenomenological attitude (further described in section 3), i.e., with reflective awareness aiming to bracket the natural attitude and thematize experience precisely as it appears. (This distinguishes SROE from methods based on examining concurrent experience within a natural attitude.)

The following points outline the basic methodological pillars of SROE (all of which will be further explained and grounded in the following sections of the article).

- Participants become co-researchers. SROE is a group-based approach requiring a small group of researchers skilled in observing and reporting on their experience and familiar with the methods of (empirical) phenomenology (Kordeš \& Demšar, 2018). This entails a radical re-examination of the standard research setting in psychological studies: the participant is not a classical psychological subject, but a principal expert in inquiring into her own consciousness, engaged in systematically and iteratively training her gesture of reflectively observing her experience and providing valid phenomenal data. (For details and further explanation, see section 4.)

- Silent meditation retreats with multiple meditation sessions per day. "Meditation" here refers to disciplined and research-oriented pursuit of being (reflectively) aware of one's ongoing experience - rather than following a specific instruction or a particular meditation technique. The general intention of researchers during meditation sessions is to reflectively observe whatever occurs in their consciousness in the present moment. Ideally, the composition of the research group remains constant throughout the series of retreats in order to enable iterative calibration of the researchers' observational activities and an in-depth investigation of chosen experiential phenomena. (For details and further explanation, see section 4.)

- Cultivating observation through collaborative exploration of observational attitude. Honing the skill of observing ongoing experience is enabled through mutual familiarization with the experience of other 
co-researchers by means of viewing video recordings of their phenomenological interviews. (See section 4.)

- Random sampling of experience during meditation sessions. During each meditation session, at a semi-random moment (e.g., leaving the first $5 \mathrm{~min}$ of the session free of sampling), the sound of the gong prompts all present researchers to describe their experience as it was unfolding immediately before the sampling moment; after taking notes, the researchers continue meditating until the completion of the session. Determining the examined moments of experience on a random basis prevents researchers from selecting (or avoiding) particular experiences that they find worthy or unworthy of examination, thereby minimizing bias.

- Phenomenological interviews about target experience. Interviews are carried out within the following $24 \mathrm{~h}$ for those samples in which experience was reflectively observed at the moment of its unfolding; see criteria (1) and (2) above. Interviews follow basic guidelines of phenomenological investigation (e.g., Depraz et al., 2003; Petitmengin, 2006); they are conducted in the form of dialogical inquiry (see section 6) among the members of the research group.

- Qualitative analysis of interview transcripts and journal entries. Acquired phenomenal data are further processed in qualitative analysis, the description of which exceeds the scope of this article (see, for instance, Petitmengin et al., 2018; Kordeš, 2016). However, the interviews and journal entries can themselves (even before the analysis) already present results of a particular (case) study, amounting to descriptions of the researchers' experiential landscapes at the moments of the sampling.

In the next four sections, we will explain and defend the most unorthodox requirements of SROE research format. We will first specify the notion of reflective awareness, and distinguish reflective observation of experience from other ways of examining experience (section 3 ). Then, we will address the issue of cultivating the attitude of reflectively observing ongoing experience and present our proposal for achieving this in a group of researchers (section 4). We will discuss the consequences of the proposed re-examination of the role of participants as principal experts on their experience - specifically with regard to the interview technique appropriate for examining reflectively observed experience (section 6). We will also address the epistemological challenges of employing SROE in the context of cognitive science and discuss a non-representationalist framework for understanding the meaning and epistemic value of the acquired data on experience (section 5).

\section{The notion of reflective observation}

\subsection{Pre-reflective and reflective awareness}

Much of contemporary literature on investigating experience not only distinguishes between the aspects of mental life that one is either conscious or unconscious of, but further differentiates between conscious (or at least not entirely unconscious) phenomena that one is aware of in an explicit, thematic, reflective way, and those that one is aware of implicitly, unthematically, or pre-reflectively. This distinction is central to the phenomenological tradition as well as some methods for acquiring empirical data on experience. Edmund Husserl, in his analysis of inner time-consciousness (1991), for instance describes the awareness of the usual flow of experience as unthematic: rather than constantly objectifying what she experiences, the experiencing person is usually unthematically aware of her experience simply by living through it in its flowing. However, one can also become thematically aware of the otherwise unnoticed pre-reflective aspects of one's experience through the act of turning towards this pre-reflective flow of consciousness in reflection, and it is only in reflective awareness that experience becomes an object of investigation:
"We must $[\ldots]$ distinguish: the prephenomenal being of experiences, their being before we have turned towards them in reflection, and their being as phenomena. When we turn towards the experience attentively and grasp it, it takes on a new mode of being; it becomes 'differentiated', 'singled out'” (Husserl, 1991, p. 132).

In the work of some authors (e.g., Thompson, 2007; Zahavi, 2019), the notion of reflective awareness of experience (or reflective self-awareness) is tied to carrying out an additional, retroactive act of reflection after the experience that is being reflected upon has already transpired (with the term pre-reflective, by contrast, referring to the kind of awareness of experience that is both logically and temporally prior to such act of reflection). Other authors (e.g., Froese et al., 2011; Petitmengin, 2007), on the other hand, also use the term reflective to denote a way of relating to one's ongoing experience. Claire Petitmengin, whose micro-phenomenological approach is based on the idea of bringing pre-reflectively lived experiences from the past to the reflective awareness of the present, describes reflective experience as "experience which is lived while being fully aware of itself or self-aware" and is as such "immediately accessible to reflective consciousness and verbal description" (Petitmengin, 2009, p. 9); pre-reflective experience, on the other hand, refers to experience "which is lived without being fully aware of itself or self-aware" and "which does not recognize itself", but might nevertheless come under the light of reflective awareness through a later micro-phenomenological interview (ibid.).

Given its importance for philosophy of mind and research in (empirical) phenomenology, the distinction between pre-reflective and reflective (as well as unthematic and thematic, implicit and explicit, etc.) awareness is surprisingly difficult to draw. The problem does not seem to stem only from differences in terminology and theoretical backgrounds, but is also apparent when one attempts to understand what the two notions would refer to in one's actual lived experience. In the present article, we are predominantly interested in the latter, using the distinction between reflective and pre-reflective awareness (or experience) not as a metaphysical-theoretical categorization of consciousness, but as referring to two different ways of what is it like to be conscious of one's ongoing experience. We use the expression "reflective" (for the lack of a better term) to denote a particular kind of attentive awareness of ongoing experience, and call the observation of ongoing experience characterized with this kind of awareness "reflective observation". ${ }^{10},{ }^{11}$ As we have shown in Kordeš et al. (2019) and will illustrate below, becoming aware of one's ongoing experience is not an all-or-nothing matter, and even real-time reflective observation of experience can be carried out with different degrees and kinds of attention.

\footnotetext{
10 Both the term "reflective" and the term "observation" fall short of capturing the experiential characteristics of becoming aware of - or coming into contact with - one's experience. When tied to the underlying mirror metaphor, the term "reflective" seems to imply a distancing from the experience in question (cf. Petitmengin, 2009); when tied to the idea of a subsequent independent act of reflecting upon (already transpired) experience, it similarly implies that the experience in question is distinct from - existing both logically and temporally prior to - the reflective act (cf. Thompson, 2007). Similarly, the term "observation" seems to presuppose a sharp distinction between the object of observation and the observational activity (Petitmengin, 2009; Zahavi, 2019). We do not commit to these presuppositions and implications and use both terms as placeholders for a particular kind of attentional activity that we will attempt to better characterize throughout the remainder of this article.

${ }^{11}$ As we will show in the next subsection, our description of reflective observation is closely tied to the notion of the phenomenological attitude. In contemplative literature, on the other hand, a similar kind of attending to ongoing experience is sometimes described as "being present in the moment", "being present with experience", or with specific notions such as "attentive presence" (Depraz et al., 2003) or "witnessing" (Thompson, 2014) - a kind of vocabulary we attempted to avoid so as to distinguish the proposed approach to investigating experience from any specific mindfulness meditation tradition.
} 
In summary: reflectively observing experience, in our usage, means attending to experience precisely as it appears in consciousness of the experiencing person. While the remainder of this section will be dedicated to the clarification of the meaning of these terms, we first ask the reader to compare the following two samples from Kordeš et al. (2019) that describe the experience of thinking.

In the first sample, the gong caught the researcher while she was absorbed in her thoughts without reflectively observing the ongoing experience; she became aware of the way in which the experience appeared (e.g., in inner speech) only after the sampling moment:

\#4 "Again in the state of being absorbed in thinking. A thought: 'This is because I don't have a washing machine at home' - and gong. [At the moment of the gong I notice that] I experienced the thought in inner speech. This is an imagined episode of what I would answer if someone asked me why I wash my dishes by hand. At the same time, I see the 'film in my head': I see my hands washing a pot." (C5-R5-D22)

In the second sample, by contrast, the gong sounded while the researcher, according to her report, was reflectively observing her experience as it was unfolding; she was attentively aware of the way in which the experience appeared (e.g., in the form of words against a black canvas) already before the sampling moment:

\#5 "In the state of attending to thinking. A thought appears: 'Why is nothing happening?' At the same time, I was realizing that this is already a thought - then, the gong. The first thought appears in the form of words against a black canvas, its meaning is more understood than read. The realization that this is already a thought appears only as a meaning, no image." (C5-R5-D2-4)

\subsection{Reflective observation and phenomenological attitude}

Many contemporary approaches to investigating experience reflect the phenomenological idea that describing experience precisely as it appears to the experiencing person requires a particular attentional shift that frees the experiencer's focus from the ties of the natural attitude (Husserl, 1983). It is only through suspending the everyday preconceptions, judgments, explanations, and theories about the world, as well as the directedness towards the content of experience (and what objects in the world this content purportedly represents), that one can adopt the so-called phenomenological attitude enabling attending to experience precisely as it appears in consciousness (compare to the so-called satellite dimensions of experience; see Petitmengin, 2006; Petitmengin et al., 2018).

However, even though it represents the fundamental method of the phenomenological approach, the experiential (as well as the philosophical) gesture of the attentional shift towards experience itself usually described in the phenomenological literature as the method of the epoche - lacks a clear definition. Some authors insist that the phenomenological epoché, as it was described by Husserl, is a strictly philosophical tool (see Zahavi, 2019); other, more research-oriented phenomenologists describe it as a method that can be employed in empirical research into human experience (see Depraz et al., 2003; Finlay, 2008; Morley, 2010). While it remains unclear just how the epoché can be carried out in research practice (especially as Husserl's published writings, focusing mainly on the results of the epoché, tend to lack sufficiently specific instructions that would make performing it accessible to an intersubjective community of researchers), various contemporary methods for investigating experience integrate the intention of putting the natural attitude out of play at the core of their methodological guidelines. ${ }^{12}$

Furthermore, it appears that the phenomenological attitude can be adopted both in real-time (i.e., while initially living through the experience of interest) or retrospectively (i.e., when examining an experience from the past). (Surprisingly, this seemingly important distinction is rarely explicitly mentioned in phenomenological literature and firstperson studies.) In methods based on retrospection, such as microphenomenology (Petitmengin, 2006) and descriptive experience sampling (Hurlburt, 2011), the phenomenological attitude is usually adopted only after the experience of interest had already been lived through, namely as the participant is remembering past experience in the context of the interview, or is writing down notes about it immediately after the moment of the sampling. While it might occur that due to chance or posterior selection, the examined experiential moment happens to be one of which the participant was reflectively aware already while living through it (e.g., various examples from Petitmengin et al., 2017; Petitmengin et al., 2009), these methods do not rely on reflectively observed experiential moments systematically. Despite its frequent mention of the phenomenological attitude, the field of first-person research thus currently appears to lack a method for which maintaining real-time phenomenological awareness would constitute a methodological premise.

Compared to similar phenomenologically inclined, but retrospection-based approaches, SROE is grounded in adopting the phenomenological attitude already at the moment of first living through the examined experience. While SROE's next steps - sampling and interview-based phenomenological examination - are similar to descriptive experience sampling and micro-phenomenology, the strategy to target experience that is reflectively lived is considerably different from the one where experience is reflectively examined only in retrospect: in the former case, the experiencing person necessarily adopts the phenomenological attitude already at the very first instance of relating to experience.

\subsection{Nuances of reflective observation}

In a subset of samples acquired in Kordeš et al. (2019), which were evaluated as reporting reflective observation, the experiential shift from the natural towards the phenomenological attitude was described in a variety of ways. In some cases, researchers reported on first relating to an aspect of experience (e.g. a thought, a particular perception or bodily sensation ...) with a habitual natural attitude, and only then shifting their way of attending to this aspect of experience towards focusing on how it was given. In other words - to use the phenomenological vocabulary - the bracketing of the natural attitude occurred after an aspect of experience had first been noticed in another, non-phenomenological sense - and often resulted in the transformation of the aspect at hand, e.g. the below example of observing the feeling of pain (\#8). In other cases, researchers reported on maintaining, at the moment of the gong, a particular kind of experiential state: an attentional disposition that they sometimes described as being open or receptive towards whatever would occur in consciousness - as if they had already bracketed the natural attitude "in advance" and were, at the moment of the sampling, disposed to reflectively attend to whatever (if anything) would emerge as the object of feeling, perception, or thought:

\#6 "Stability, firmness, and peacefulness. Focus on breath and the moving of the belly; in the background, the feeling of the body, clear

\footnotetext{
$\overline{12}$ While some of them do so explicitly (e.g., Depraz, 1999; Depraz et al., 2003; Giorgi, 2009; Morley, 2010; Petitmengin, 2006; Vermersch, 1994), others (e.g., Hurlburt, 2011) merely agree that what they are endeavoring towards is in line with the general idea of phenomenology.
} 
and steady. There are no thoughts; in a longer period before the gong (throughout the meditation), a few commentaries appeared from time to time in the background - I did not untangle them, but only observe - they fainted very quickly." (C3-R4-D1-8)

These observations match the remarks from phenomenological literature that discuss phenomenological attitude as a continuous achievement and describe epoché as a incessantly reactivated activity of suspending judgment, rather than an on-and-off switch into a different attentional state (Depraz, 1999; Morley, 2010).

\subsection{Reflective observation and metacognitive awareness}

By now, it should be clear that the notion of reflective observation does not refer to any kind of attending to the ongoing experience. In particular, we want to draw a distinction between being reflectively present with the ongoing experience in the phenomenological sense, and paying attention to elements of experience in other ways - for instance by thinking about them, analyzing or attempting to explain them, categorizing them, etc. Consider the following sample catching the researcher while she was paying attention to her experience of pain:

\#7 "I was observing the pain in my back, pain in my left knee, pain in the lower part of my belly, and my breath. I was thinking about the reasons for the pain in the lower part of my belly, even though I didn't verbalize these thoughts in my mind. I just got the feeling that with every exhale, I feel the pain in my ovaries. Then I somehow rationalized that the reason for the pain is the full bladder." (C7-R4D1-9)

This example of attending to the ongoing experience of pain through searching for reasons that could be causing it serves as a good illustration of what we do not mean by reflective observation and reflective awareness. While it may, in some cases, be encompassed under some (broader) definitions of what psychological and philosophical literature refers to as metacognitive awareness or meta-awareness (cf. Schooler \& Schreiber, 2004), reflectively observing experience (in our usage) emphatically does not refer to metacognitive awareness in the sense of thinking about or cognitively monitoring ongoing experience (such as \#7). ${ }^{13}$

For illustration, compare the above sample to the following one, in which the researcher, rather than thinking about or attempting to conceptualize the ongoing experience of pain, simply becomes aware of it:

\#8 "My focus was on the bodily feelings. My leg started to hurt again, so I began to observe this pain. At first, I felt the need to move my leg, and then I consciously focused my attention on this need, and pain suddenly became just another feeling. The painful part disappeared." (C5-R3-D1-8)

Experientially, reflective observation - compared to analyzing, monitoring, or thinking about experience - is not felt as a separate observational activity aiming at the observed experiential phenomenon from the outside. When being reflectively observed, the (aspect of) experience is perhaps best described as being enveloped with (rather than looked at by) reflective awareness, in the sense that reflective awareness

\footnotetext{
13 One might be engaged in analyzing, monitoring, or thinking about experience and simultaneously observe this activity with reflective awareness. Consider the following sample from our research: \#15 "I was present - aware of the content of my thoughts and the fact that I was thinking. I was observing the 'buzzing in the room'. A thought appears: 'Is the buzzing real, or am I only imagining it?' Awareness of thoughts, feelings, stimuli ... A part of me is trying to analyze them (e.g., find out where the buzzing is coming from), while I at the same time don't lose the presence with experience, I don't get submerged into it." (C5-R5-D1-4).
}

becomes an experiential feature of the observed phenomenon. ${ }^{14}$

\section{Cultivating the skill of reflective observation}

The samples acquired in the course of Kordeš et al., 2019 demonstrate that the attitude of phenomenologically observing ongoing experience can be present in different forms and to different degrees, and that the attentional shift from the natural attitude towards the way experience is given is not an on-and-off switch. While it might sometimes come into place suddenly and without a systematic endeavor, pursuing the phenomenological attitude as the "instrument of observation" for consciousness research cannot rely on such spontaneous events, but requires a disciplined and persistent cultivation. Rather than following a prescribed observational instruction or meditation technique, such cultivation has to be based on each individual researcher's gradually becoming aware - and consequently honing - her patterns of relating to her own experience.

\subsection{Reflectively observing experience and mindfulness meditation techniques}

In recent decades, there have been a number of discussions comparing the method of the epoché to different Eastern practices such as yoga (Morley, 2001) and Samatha-Vipassanā meditation (Depraz, 1999; Depraz et al., 2003; Kordeš \& Markič, 2016; Varela et al., 1991). Some authors have for example suggested that classical Buddhist meditation techniques such as those belonging to the Samatha tradition might be the most appropriate candidate for the "telescope" for consciousness research (e.g., Wallace, 1999). The aim of this article is not to participate in the theoretical debate about the similarity of meditation techniques and phenomenological guidelines (a debate that suggests that concepts such as mindfulness, nonjudgmental attending, and epoché might not be defined clearly enough to be operationalized in the context of scientific research). Nevertheless, we must acknowledge that the observational attitude sought in SROE - attempting to attentively observe ongoing experience with reflective awareness - closely resembles the standard definition of mindfulness (e.g., Kabat-Zinn, 1994). Despite this similarity, the proposed approach to investigating experience is emphatically not based on following any specific mindfulness meditation school. The following paragraphs present the main reasons why we decided not to rely on a particular meditation technique.

- Bracketing presuppositions with regard to meditation. The common rationale behind basing research into experience on practitioners that have dedicated their lives to pursuing a particular kind of meditation technique is that such highly experienced meditators are able to provide more reliable and valid reports on their consciousness. While this might be the case, there has as of yet been no systematic research that would indicate which (if any) of many possible types of meditation would be the most appropriate to select for the purpose of empirical investigation of consciousness.

\footnotetext{
14 The distinction between reflectively observing and analyzing or conceptualizing experience can be linked to the distinction between different attentional dispositions (Petitmengin et al., 2009). In their study of the experience of listening to a sound, Petitmengin and colleagues describe three different types of auditory experience as resulting from three different modes of participants' attending to the sound. Namely, in listening to a sound, participants directed their attention either a) towards the event at the source of the sound, b) towards the sound "in itself", or c) towards the way in which the sound was being felt. The latter type of attentional disposition most closely resembles the attitude of reflective observation. (See also different attitudes adopted in relating to one's experience of pain in section 5 , footnote 16, and various examples from Kordeš et al., 2019).
} 
- Equal accessibility for researchers. Our ambition in developing SROE was to establish a format of research that could be practically implemented in the context of cognitive science and open to a diverse set of researchers interested in and committed to exploring their consciousness. Being trained in a particular type of meditation practice or being a long-term follower of a specific meditation school should not be a requirement for participation.

- Self-regulation mechanism. One of the main pillars of scientific research is the capacity for self-regulation, by which scientific methods, models, and theories are assessed and revised on the basis of their success in describing or explaining their domain of investigation. Exploring consciousness through standard meditation techniques, by contrast, does not abide by any such self-regulatory apparatus. Observations that diverge from what is described in the meditation canon are usually seen as stemming from the inadequacy of the practitioner's way of practice, rather than regarded as potential discoveries that could inform or challenge the already existing knowledge.

- Orientation towards research. The aim of the majority of approaches to meditation is to achieve particular experiential states - deemed to reflect the appropriate way of practicing - rather than to pursue an open-ended approach to investigating consciousness. This tendency showed up in numerous samples from Kordeš et al. (2019). In a number of samples categorized as Presence, the researchers, rather than remaining open to nonjudgmentally observing whatever would appear in consciousness, described a kind of attachment to their current meditative state or a goal-oriented pursuit of a desired one. Consider this excerpt:

\#9 "Very present with an intensely pleasant feeling. Everything there is, is the breath and an immersion in pleasant nothingness. No images. Strong intention to stay in this state." (C4-R4-D2-1)

In reports like this one, the researcher could be said to be in contact with experience; however, the reported attitude does not present an ideal ground for acquiring phenomenal data, as the researcher's pursuit of a particular meditative state might narrow down the possibilities of observing whatever appears - and how it appears - in her consciousness. For research purposes, a more appropriate attitude is one that combines the phenomenological contact with ongoing experience with a critical and persistently curious openness, enabling the researcher to remain open to novel, perhaps surprising aspects of experience, rather than getting stuck in a particular "local minimum" of pleasant and comfortable feelings.

In addition to the other two criteria for the kind of experience targeted in SROE, i.e., experience that (1) is being attended to at the moment of its unfolding and (2) is being attended to with a phenomenological attitude, the proposed approach to cultivating reflective observing therefore also requires another condition: letting go of the goal-oriented attitude to promote a research-oriented, curious, and open witnessing to whatever appears in consciousness. ${ }^{15}$ Attending to experience with this attitude is not a soteriological endeavor. It does not promise a goal in the sense of a therapeutic release or a blissful experiential state. Such events are of course not excluded - in the meditation study reported in Kordeš et al. (2019), they indeed turned out to be quite frequent; however, the research-oriented attitude requires the researcher to continually put them in brackets (together with other beliefs and convictions) so as to be able to pursue new findings about experience.

\footnotetext{
15 There exist various meditation techniques that aim at the cultivation of attitude very similar to the one we describe here and which one might consider "borrowing" for the purpose of phenomenological investigation in the context of cognitive science; however, there are no a priori - or, as of yet, evidencebased - reasons for choosing a particular technique over the other.
}

\subsection{Cultivating observational attitude, not meditation technique}

Instead of relying on techniques from any specific meditation school, we decided to base SROE on an approach to observing consciousness that would satisfy the above four requirements (i.e., bracketing presuppositions of any particular meditation tradition, accessibility for a diverse range of consciousness researchers, possibility of self-regulation, and orientation towards research) and that would be defined narrowly enough to allow for replication by other research groups.

Our experimentation with instructions for observing experience in the meditation study (Kordeš et al., 2019) indicated that a unified directive cannot guarantee that researchers will attend to their experience in a comparable way. At the first retreat of the study, we attempted to summarize the idea of the phenomenological examination of consciousness by extensively discussing phenomenological guidelines and asking the researchers to simply "non-judgmentally observe consciousness as it appears". Even though all researchers were familiar with the notion of the epoché and had a sense that they understood the instruction, we were surprised to see that this simple directive led individual meditators to perform remarkably different attentional gestures. What they were doing and experiencing during meditation turned out to vary and to have much to do with individual researchers' prior meditation experience, their individual attentional habits and observational interests, their ideas about what aspects of their consciousness are worth observing and how to turn their attention towards them.

Seeing that neither agreeing on a propositional instruction nor prescribing a particular technique (cf. Kordeš et al., 2019) ensured unified approach to attending to experience, and recognizing the need to enable, for each individual researcher, the conditions for developing an intimate understanding and practice of reflectively observing their own experience, we decided to base the cultivation of the target observational attitude on a different approach: on honing individual researchers' gestures of observation through a gradual alignment of researchers' observational gestures over the course of the retreats.

The approach we adopted in the meditation study and propose as the methodological foundation for SROE is grounded in two central elements.

- Simple and well-articulated research guideline for the target observational attitude: "Be reflectively aware of whatever occurs in consciousness in the present moment".

Articulating and deciding to follow this guideline is not enough for ensuring the desired (and comparable) observational attitude in all researchers. However, it can serve as an anchor, presenting for a researcher a starting point for each meditation session, as well as reminding her to check, during meditation, whether she is indeed radically pursuing the phenomenological attitude - that is, whether she is attending to all the aspects of the current experiential field, rather than merely following her intuitions of what it means to observe experience. As an example, consider the following excerpt:

\#10 "This state was really pleasant, although it demanded an effort to maintain it. I was slightly afraid that it would go away." (C4-R5D3-1)

Had the researcher, at the moment of sampling, radically pursued the phenomenological attitude, she might be prompted to broaden her attention to observe, for instance, the experience of effort in maintaining the state, the experience of being afraid, the experience of being the subject of these two feelings, etc. The goal of following and returning to the guideline is to gradually transform and refine observational habits of this direction, cultivating the gesture of nonjudgmental attending to the entirety of the experiential landscape, and at least partially countering the habitual pull towards the natural attitude. 
- Intersubjective alignment of observational attitudes within a group of researchers.

As described in section 1, the process of cultivating the observational attitude in Kordeš et al. (2019) was based on deepening individual researchers' understanding and practice of phenomenological examination through iterative intersubjective testing and alignment with other members of the group. This was achieved through enabling all researchers to get acquainted with the experiential reports of their colleagues via viewing the video recordings of others' phenomenological interviews, making it possible for them to inform and expand their own observational practice. Each researcher viewed the recordings of others alone, on his or her own, without any determined goal (apart from learning about others' experience and investigations during the meditation session). In light of its encouragement of collaborative exploration of phenomenological examination, the proposed format enables the members of the group to gain access to a systematic dialogical inquiry into experience of their peers, but avoids the group dynamics of typical meditation "sharings" (e.g., peer pressure) that can encourage selective reporting, rationalization, and uncritical interpretations of meaning. As demonstrated in Kordeš et al. (2019; see especially Figure 16, p. 224), this process can lead towards an eventual alignment of different researchers' ways of turning towards their experience, without following any predefined instruction, technique, or definition of phenomenological examination or meditation. ${ }^{16}$

\section{Epistemological characteristics of acquiring phenomenal data in SROE}

\subsection{Excavation fallacy: disturbing the experience by observing it}

There is a long history of objections to the possibility of the scientific investigation of lived experience from both the scientific and philosophical community (Comte, 1830; Searle, 1992; Dennett, 1993; Metzinger, 2004; for an overview, see Varela, 1996; Bitbol \& Petitmengin, 2013). As the examined experience is accessible only from the first-person point of view of the experiencing person, and in addition does not present a stable object of examination that would remain unchanged over time and be easily explored anew, there is no intersubjective or even intersituational approach to testing and validating the acquired phenomenal data (see Kordeš \& Demšar, 2018; 2019).

The challenge of devising a method for acquiring empirical data on lived experience (i.e., phenomenal data; Varela \& Shear, 1999) dates back to the very beginning of psychology and consciousness science. From the development of early introspectionist schools and the emergence of the phenomenological approach at the beginning of the 20th century to contemporary approaches such as think-aloud protocols, experience sampling, and micro-phenomenology, different attempts at establishing a method for empirical investigation of lived experience have been met with a host of epistemological and methodological difficulties. The most prominent among these revolve around the question of the epistemic value of the act of examining experience, and the meaning of acquired results. What kind - and what degree - of epistemic access do we have to our experience? Can any method claim to provide data on undisturbed, unspoiled experience - considering that every attempt at observing consciousness seems to alter or interfere with the very conscious state that one is trying to observe? What do we, in fact, examine when we claim to examine consciousness?

\footnotetext{
${ }^{16}$ For any research group intending to conduct a study using SROE research format, it is important to emphasize that such convergence of individuals' approaches to phenomenological examination cannot be guaranteed, but rather may (or may not) occur through a spontaneous and self-organized process based on researchers' mutual familiarization with one another's (investigations of) experience.
}

Regardless of the method employed for collecting reports on experience, once the experience has been thematized, there is no way of independently evaluating whether the acts of observation and reporting constructed the result or whether they merely retrieved information about experience. In the absence of the possibility of intersubjective and intersituational corroboration of acquired data, how can one make sure that the acquired data are results of examining - rather than disturbing or even inventing - the examined experience? It appears that answering to this challenge, sometimes dubbed the excavation fallacy (see Introduction), has the potential of either grounding or renouncing the soundness of not only the approach proposed in this article, but of any attempt at the acquisition of empirical data on experience.

\subsection{The intrinsic link between the way of observing and the resulting experience}

\section{Consider the following example}

\#11 'I'm sitting in Lepena, attending to my bodily sensations. Then, everything around me and behind me starts to 'disappear', to be enveloped in darkness: who are we, the people sitting in Lepena, where we're sitting, etc. There is only me and the way I'm sitting (aware of my body as a whole) and the way I'm present with what is happening - the feeling of transitioning towards a very beautiful state of peace. In the background of experience, I feel the expectation and curiosity about this darkness and whether anything is going to appear - this is the experience that is interrupted by the gong." (C3R5-D4-3)

As any kind of attending to experience, also reflective observation does something to the observed experience of those that cultivate it. But what is this something - does it change experience, or does it change the act of observation? Perhaps both? There are various potential "sources" of the difference in reports on experience provided by contemplative phenomenologists or meditation practitioners more broadly:

a) Training in meditation or contemplative phenomenology can, using William James' metaphor, "lower the threshold of consciousness" (cf. Sparby, 2015), enabling researchers in the states of meditative presence to observe consciousness more precisely and truthfully closer to the way it really is. Meditation here is seen as a way of clearing the lens of the telescope for consciousness research (e.g., Wallace, 1999).

b) Cultivating a particular attentional disposition (see Petitmengin et al., 2009) towards their consciousness, the meditators - rather than increasing their overall sensitivity to consciousness, as suggested in (a) - learn to observe their experience in a different way and focus on different aspects of consciousness. They, for instance, train to redirect their attention from more superficial elements such as thoughts and mental imagery towards more fundamental perhaps even transcendental - structures of experience.

c) The cultivation of reflective attending, rather than cleaning the lens of the observational instrument (option a) or adjusting its focus (option b), transforms the domain of observation itself. In other words, the difference in meditators' observations and reports stems not from their increased or changed sensitivity to their experiential field, but from the changes of the very experiential field that they are trying to observe.

How could one determine which of these three options is correct - or if settling for a possibility of a mixture of all three (e.g., that cultivating the gesture of observing experience might at the same time change the experiential field of the observer and influence her focus and precision in 
observing experience), how could one determine the relative contribution of each of them? We maintain, and have argued elsewhere (Kordeš \& Demšar, 2018, 2019), that these questions cannot be answered on the basis of empirical data - that is, without engaging in ontological speculations about experience that go beyond what can be empirically determined. Due to the inherent impossibility of separating between the process of examining and the domain of examination in study of lived experience, the field of first-person research cannot consistently adopt a "view from nowhere", regarding its object of examination (i.e., the examined experience) as an entity whose existence and structure are independent of the act of examination (Kordeš \& Demšar, 2019). As put by Varela (1996, p. 14), "[t]o speak of experience as being standard, raw, or pure generally makes no sense. All we have is experience at its own level of examination, and depending on the kinds of effort and methods brought into play."

In line with these considerations, we suggest that any method for examining lived experience - including the approach we propose here must acknowledge that the act of observing (and reporting) experience does not merely retrieve information from an independent, ideally undisturbed stream of consciousness, but constructively contributes to the generated phenomenal data. ${ }^{17}$

\subsection{Horizon of attending to experience as an element of experience}

Conceiving phenomenal data not as descriptions of a domain existing independently of the acts of examination, but as descriptions of this domain's reactions to particular ways of probing brings to an important consideration: the way in which one examines their experience plays a crucial role in what ends up being reported as a result of examination. Therefore, in order to understand what we see in experience (i.e., the acquired phenomenal data), we must understand the perspective of looking at it (i.e., the way of acquiring data, from the first attentional turn towards the experiential field to generating the description). The horizon of examining experience (as defined in Kordeš \& Demšar, 2018, 2019; Kordeš et al., 2019) includes the totality of factors that can affect (and indeed, co-determine) the final report on experience - or, even further, the final results of an empirical-phenomenological study (such as the constructed categories of experience, the posited relationships between them, and/or the uncovered generic structures of experience). These factors span multiple steps in the production of the final report on experience: everything from how experience is first grasped and gradually thematized by the individual researcher (starting with the attitude with which she turns towards her yet undifferentiated experiential stream), through how it is conceptualized and verbalized when describing it in notes or in the context of an interview (including the course of the interview process, the relationship with the interviewer, etc.), to how the generated report is later analyzed, validated, and assessed in a community of researchers. ${ }^{18}$

Despite the multitude of factors co-determining the way of inquiring into experience, spanning the entire research process, the step that appears to most substantially shape the results of examination is the first

\footnotetext{
17 Elsewhere (e.g., Kordeš \& Demšar, 2018), we describe the act of forming knowledge about experience borrowing the constructivist notion of enaction (Varela et al., 1991) in order to stress the mutual specification of the observing and the observed in examining experience. To say that first-person knowledge is enacted (rather than retrieved or constructed) allows for a "middle path" in the dilemma of whether the results of such examination are homomorphically recovered from the pristine, independently existing experience, or arbitrarily invented by the person describing their experience.

18 On a larger scale, horizons of examining experience are also shaped by the research questions and aims of the particular study, as well as the theoretical background that contributed to its design, and various elements of its social environment (which psychology and cognitive science have usually encompassed with notions, such as peer pressure and demand characteristics; cf. Orne, 1962; Nichols \& Maner, 2008).
}

one: the initial act of turning towards experience. What we mean with the horizon of this initial turning towards can be illustrated with the following examples of random experience samples from Kordeš et al. (2019), all of which caught researchers while they were maintaining particular (different) attitudes of relating to the sensation of pain:

\section{\#12 [Attitude: Avoiding the sensation of pain]}

“'Oh, not again!' I'm dealing with the pain in my knees. Actually, I don't want to deal with it. I avoid it - there is restlessness and the desire to move." (C4-R4-D2-8)

\#13 [Attitude: Attempting to use meditation as a tool for alleviating the sensation of pain]

"I was trying to accept the feeling of the meditative posture. I tried to let myself loose into the pain - ease into the feeling of unease. There is a sense that relaxing into my body will lead to me embracing the entire experiential field." (C1-R5-D3-4)

\section{\#14 [Attitude: Reflectively observing the sensation of pain]}

"My focus was on the bodily feelings. My leg started to hurt again, so I began to observe this pain. At first, I felt the need to move my leg, and then I consciously focused my attention on this need, and pain suddenly became just another feeling. The painful part disappeared." (C5-R3-D1-8)

If the way of examining (and more narrowly, attending to) experience co-determines what is observed, detecting and articulating the horizon of this examination appears to be crucial for understanding the meaning and epistemic value of the acquired phenomenal data. As we have seen, the horizon can be shaped by a long list of factors, and explicating all of them appears to be a nearly impossible endeavor. However, as phenomenologists, we can access the horizon in a more direct way: it can be experienced. Amounting itself to an element of experience - even if a highly subtle one - the horizon can be examined as any other part of the experiential field: not with regard to the factors and motives involved in its formation, but through turning our phenomenological focus towards its mode of appearing. We only need an angle of examination that allows the horizon to be directly noticed as a part and parcel of experience.

While we maintain that every kind of attending to experience (phenomenological or not) necessarily entails a horizon of one's relation towards their experiential field, an important advantage of SROE is that it has the potential to observe this horizon at the moment of the unfolding of experience. Compared to other similar approaches to examining experience, SROE is specifically and systematically grounded in paying concurrent reflective attention to the first moment of turning towards experience, thereby enabling an insight into and description of the initial gesture through which the data was obtained.

Acknowledging the horizon of turning towards experience (including elements such as the attitude of attending to the experiential field, attentional and focusing activities involved in attending to it, and the related subtle dimensions of experiences, such as anticipatory background feelings) as itself amounting to an important part of experience requires accepting all these elements as necessary components of the eventual phenomenological description. The natural attitude might make it seem that lived experience resembles a landscape, filled with experiential "objects" which may be described through analogies to photographs or films. With its ongoing endeavor at bracketing the natural attitude, SROE offers a possibility of a richer, more exhaustive description: what, at the first glance, might have appeared to be passively perceived, can be recognized as being continuously 
constructed. In this way, how one relates to one's ongoing experience can be examined as an important part of the experiential field, with the description of gestures involved in one's attending to experience consequently coming to form crucial elements of the generated phenomenal data.

\section{Phenomenological interview as a dialogical investigation}

We do not claim that the kind of reflective observation that we propose circumvents the constructive dimension of examining experience, leading the researcher towards seeing experience in its pristine, undisturbed form. However, reflective observation does allow for the first notion of examined experience to take shape already in the moment of the unfolding of experience. Shaped in the first moment of turning towards the ongoing experience, we maintain that experience that has been reflectively observed simultaneously with its unfolding is more resilient (although far from immune) to the constructive potential of the further steps of its thematization. This enables the kind of the interview technique that we propose for SROE to take - in comparison to other similar methods - a more critical, dialogue-based approach to phenomenological inquiry.

Standard retrospective methods for studying experience put a strong emphasis on attempting to minimize the constructive role of the phenomenological interview in the generation of phenomenal data. Micro-phenomenology, for instance, insists on using "non-inducive" questions that avoid leading the interviewee towards reporting on elements of experience partially determined by the interviewer (Petitmengin, 2006). Similarly, the expositional interview in descriptive experience sampling aims at using "open-beginninged" questions (Hurlburt, 2011) that attempt to bracket all presuppositions about what - if anything - the interviewee might have been experiencing at the sampling moment.

The phenomenological interview technique that we propose for SROE is envisioned as a dialogical investigation, rather than a careful and non-inducive guidance of the interviewed subject in exploring her experience. While sharing many of the characteristics of abovementioned interview techniques, it puts less emphasis on the caution involved in choosing and articulating particular questions, allowing for a greater freedom of phenomenological investigation (especially in comparison to the micro-phenomenological interview method).

Another feature of SROE that allows for this approach to interviewing is that the interviewee is herself a fully-fledged researcher. The interview can be described as a process of collaborative dialogical investigation along the lines of participatory sense-making (cf. De Jaegher \& Di Paolo, 2007), carried out by two equal co-researchers (cf. Kordeš, 2016). ${ }^{19}$ In the context of SROE, both the interviewer and the interviewee intimately understand the phenomenological notions of the epoché and phenomenological attitude, and report on their experience with the explicit aim of acquiring genuine and precise phenomenal data. In this research situation, the role of the interviewer is to provide critical assistance in the interviewee's investigation of her sampled experience. This assistance can take the form of standard phenomenological guidance into the experiential inquiry, but can involve posing questions or prompts aimed at expanding the interviewee's space of awareness, indications of inconsistencies or gaps in the generated report, requests for clarifications and re-examinations, as well as explicit inquiries about potentially present, but not yet described elements of examined

\footnotetext{
19 While the concept of co-researchers is recognized by both the microphenomenological method and - to a larger extent - descriptive experience sampling, SROE entails an even more radical revision of the classical understanding of the roles of the participant and the researcher in psychological research, requiring the person whose experience is being investigated to be skilled in empirical phenomenology and carry the larger part of the responsibility for the richness and validity of acquired phenomenal data.
}

experience (e.g., sense of self, existential feelings, attentional disposition, etc.).

\section{Applications of SROE research format}

The question of the reliability of the proposed approach will be addressed only after other, independent studies will be carried which will employ the proposed research design. So far, our observations, partially described in Kordeš et al. (2019), indicate that persistent cultivation of one's own experience shows the potential for providing surprisingly replicable findings (preliminary findings on some phenomena, for instance the experience of gist, see Kordeš et al., 2019, and a subset of experiences of thinking, have already been replicated in later retreats and by different researchers). This observation is in line with findings from other fields in qualitative research, where it often turns out that in-depth results, even when acquired with a small and specific sample of participants, can bear higher research relevance than a superficial overview of a bigger sample (e.g., Flyvbjerg, 2006).

With regard to the scope and limitations of the proposed approach and its position within the field of first-person research, we emphatically do not want to present SROE as a replacement for any of the established methods for examining experience, or suggest that it would solve all challenges to acquiring phenomenal data (or even the excavation fallacy in particular). Indeed, focusing only on moments of experience that were lived through with a reflective attitude might impede the investigation of certain phenomena, which are otherwise readily accessible to other approaches (for example the "frequently occurring phenomena of experience" surveyed by the descriptive experience sampling method; cf. Heavey \& Hurlburt, 2008).

Additionally, the execution of SROE might be more complicated and demanding compared to existing methods with well-established protocols (e.g., Petitmengin, 2006; Hurlburt, 2011; Ericsson \& Simon, 1993). Since, as described above, the success of the research format relies on the participatory cultivation of the phenomenological attitude, SROE cannot be based on following a predefined protocol. In fact, any research project grounded in SROE requires a longitudinal collaborative work of a group or researchers, who repeatedly meet in order to investigate their experience. These meetings include the organization and enactment of meditation sessions (and/or entire retreats), the organized sampling of these sessions, conducting interviews for the target samples in which the interviewee was attentively aware of her ongoing experience, viewing recordings of one another's interviews, as well as intermittent evaluation sessions and preparatory sessions in which members of the research group collaboratively evaluate the ongoing challenges and findings as well as plan the upcoming steps in the study.

In order to collaboratively examine and refine the observational attitude and activity, studies grounded in SROE require a longitudinal and iterative research design, based on repeating research cycles of the following structure: (sampled) meditation session - phenomenological interviews about selected samples - intersubjective sharing of phenomenological reports - analysis of phenomenological reports - evaluation - planning - new (sampled) meditation session. Since the sampling of meditation sessions must remain random, a considerable number of sessions are required to collect enough data on moments in which researchers were reflectively aware of their ongoing experience (and/or were experiencing any other target phenomenon of interest; see footnote 21).

However, the demanding design of SROE studies enables, to a greater extent than any other existing approach in first-person research, answering Varela's call for a research community allowing cognitive scientists to "attain a level of mastery in phenomenological examination" (Varela, 1996, pp. 346-347). What is more, in SROE, the 
researchers do not only use phenomenological examination, but also investigate it. Consequently, what counts as phenomenological examination can therefore itself become articulated, refined, and internalized through their collaborative investigation.

\subsection{Possible lines of research}

SROE research format may prove especially useful in addressing some research questions that are less available - or available from a different angle - for other existing methods for examining experience. In particular, it can systematically target experiential phenomena that other approaches either overlook or have low probability observing in real time. In what follows, we identify a few types of experiences for which investigation based on SROE research format could be particularly suitable. ${ }^{20}$

Spontaneously emerging experiential phenomena. With a sufficiently large collection of samples, the approach based on random sampling of experience opens a phenomenological window onto the types of experiences that are difficult to elicit (or cannot even in principle be elicited; cf. Hurlburt et al., 2016) with standard task-based research paradigms in cognitive science, for instance the experience of mind-wandering (As we have shown in Kordeš et al., 2019, it is possible to acquire samples in which the researcher's mind is wandering, while she nevertheless keeps reflectively observing her experience.).

Meditation-related experiences. These might encompass phenomena typically described in meditation literature as the considerably rare experiential states that meditators are frequently seeking to achieve (cf. Kordeš et al., 2019). While research in meditation experience is currently on the rise, participants in typical contemplative studies are long-term meditation practitioners with little expertise in phenomenological examination. The rare occurrence of mystical experiences and other 'peak moments' of meditation might be difficult to catch with the first few random samples. However, a longitudinal research design with many repeated meditation retreats could increase the incidence of such phenomena. The advantage of SROE is that, when such a phenomenon is caught, it will be caught with a systematic research instrument.

Subtle aspects of consciousness that are usually hidden in the background of experience, such as felt sense (Gendlin, 1996), or existential feelings (Ratcliffe, 2005).

"Underlying" invariant structures of experience. In line with some authors' idea that practicing specific ways of attending to experience can "lower the threshold of consciousness" (e.g., Petitmengin, 2007; Sparby, 2015), SROE can enable the investigation of experiential structures that are often thought to underlie the majority of everyday experience - but that usually remain unnoticed against the foreground of more explicit contents of awareness. In our own research (Kordeš et al., 2019), we successfully applied the methodological framework that served as a precursor to SROE to investigating subtle experiential phenomena such as the pull towards mind-wandering, or the distinction between the transmodally experienced felt meaning of thinking (cf. Petitmengin, 2007) and the "illustration" of this meaning in inner seeing, hearing, or

\footnotetext{
20 This list is not meant to exclude other approaches to examining experience: some of the listed experiential phenomena can be - and/or have already been successfully tackled with other existing research methods. Spontaneously emerging experiences, such as mind-wandering, for instance, have been studied both by descriptive experience sampling (Fernyhough et al., 2018) and micro-phenomenology (Petitmengin et al., 2017), and micro-phenomenology in particular has been notably used to study meditation-related experiences (Petitmengin et al., 2017), as well as subtle and potentially "underlying" experiential structures such as the "source dimension" (Petitmengin, 2007). The advantage of (also) researching these phenomena with SROE is that with SROE, the researcher does not have to rely on the luck involved in the sample randomly catching the phenomenon of interest (like in descriptive experience sampling) or on selecting (accompanied with potential selection bias) a specific experience from the past.
}

speech (cf. Heavey \& Hurlburt, 2008). ${ }^{21}$

As we mentioned above, we remain agnostic towards the question whether noticing such structures means that the researcher became more sensitive to a dimension of experience that was already there, or that her practicing changed the very experiential field she is now aiming to observe (or perhaps a mixture of both). While our empirical observations show that in these cases, researchers feel that the former is the case (i.e., that they are uncovering dimensions that already were there, and that their practicing indeed lowered their threshold of consciousness), this feeling need not imply claims to the ontological substrate of experience. Rather, understanding SROE as a psychological instrument can go hand in hand with bracketing such ontological questions (as they, in any case, cannot be answered empirically; cf. Kordeš \& Demšar, 2019). Rather, the potentially underlying structures of experience can be simply (and without ontological commitments) re-described as those that turn out to be more stable - that is, more intersubjectively and intersituationally invariant - in response to reflective observation.

\subsection{Drawing a more complete map of experience}

The capacity of SROE for observing the first moment of thematization - and shedding light on the horizon of attending to experience might allow the researchers themselves to study the characteristics of their gestures of reflective observation. This strategy might present a way of increasing the reproducibility of data acquisition through articulating the characteristics of attentional observational activities as well as enabling their further refinement. More importantly, the ability to notice not only the "objects" populating the experiential landscape, but also the attentional activities related to the emergence of these very "objects", allows for a more exhaustive description of the field of experience - with the resulting map of this field including, among other elements, the gestures of turning towards particular aspects of experience and determining the horizon through which this turning towards is taking place.

In developing SROE research format, we drew upon research from the areas of contemplative and phenomenological studies and empirical first-person research. Inversely, we think that SROE can present a valuable tool for researchers from all these areas, recently integrated into the emerging field of contemplative phenomenology (Sparby, 2015). The formation of this field shows that philosophy and the sciences of the mind might be gradually realizing that it makes sense despite the number of epistemological and methodological challenges entailed in this endeavor - to do (rather than merely think) research based on a disciplined cultivation of reflection, and empirically assess the soundness of such investigation. If we are interested in consciousness as it appears, first-person access to experience is, in the end, all that we can start from.

\section{Author statement}

Urban Kordeš: Conceptualization, Methodology, Investigation, Writing - Original Draft, Writing - Review \& Editing

Ema Demšar: Conceptualization, Methodology, Investigation, Writing - Original Draft, Writing - Review \& Editing

\footnotetext{
21 For these research questions, the "target" experience was not, by contrast to the research format described in this article, exclusively the experience that had been originally lived through with reflective awareness, but a particular mindwandering-related phenomenon that became discernible - and amenable to investigation - only after having collected a considerable number of samples (see Kordeš et al., 2019). This illustrates how SROE can, provided the sufficiently large collection of relevant samples, be adapted and applied to research questions that do not require the target experience to have been reflectively observed.
} 


\section{References}

Bitbol, M., \& Petitmengin, C. (2013). A defense of introspection from within. Constructivist Foundations, 8(3), 269-279.

Comte, A. (1830). Cours de philosophie positive. Paris: Borrani et Droz, Libraires. De Jaegher, H., \& Di Paolo, E. (2007). Participatory sense-making: An enactive approach to social cognition. Phenomenology and the Cognitive Sciences, 6(4), 485-507.

Dennett, D. C. (1993). Consciousness explained. London: Penguin UK.

Depraz, N. (1999). The phenomenological reduction as praxis. Journal of Consciousness Studies, 6, 95-110.

Depraz, N., Varela, F. J., \& Vermersch, P. (2003). On becoming aware: A pragmatics of experiencing. Amsterdam: John Benjamins.

Ericsson, A. (2003). Valid and non-reactive verbalization of thoughts during performance of tasks towards a solution to the central problems of introspection as a source of scientific data. Journal of Consciousness Studies, 10(9-10), 1-18.

Ericsson, K. A., \& Simon, H. A. (1993). Protocol analysis: Verbal reports as data. Cambridge MA: MIT Press.

Fernyhough, C., Alderson-Day, B., Hurlburt, R. T., \& Kühn, S. (2018). Investigating multiple streams of consciousness: Using descriptive experience sampling to explore internally and externally directed streams of thought. Frontiers in Human Neuroscience, 12, 494.

Finlay, L. (2008). A dance between the reduction and reflexivity: Explicating the" phenomenological psychological attitude. Journal of Phenomenological Psychology, 39 (1), 1-32.

Flyvbjerg, B. (2006). Five misunderstandings about case-study research. Qualitative Inquiry, 12(2), 219-245.

Froese, T., Gould, C., \& Seth, A. K. (2011). Validating and calibrating first-and secondperson methods in the science of consciousness. Journal of Consciousness Studies, 18 (2), 38.

Gendlin, E. T. (1996). Focusing-oriented psychotherapy: A manual of the experiential method. New York: Guildford Press.

Giorgi, A. (2009). The descriptive phenomenological method in psychology: A modified husserlian approach. Duquesne University Press.

Heavey, C. L., \& Hurlburt, R. T. (2008). The phenomena of inner experience. Consciousness and Cognition, 17(3), 798-810.

Hurlburt, R. T. (2011). Investigating pristine inner experience: Moments of truth. Cambridge: Cambridge University Press.

Hurlburt, R. T., Alderson-Day, B., Kühn, S., \& Fernyhough, C. (2016). Exploring the ecological validity of thinking on demand: Neural correlates of elicited vs. spontaneously occurring inner speech. PloS One, 11(2).

Husserl, E. (1983). Ideas pertaining to a pure phenomenology and phenomenological philosophy (F. Kersten, trans.). The Hague: Martinus Nijhoff.

Husserl, E. (1991). On the phenomenology of the consciousness of internal time (1893-1917). Dordrecht: Kluwer Academic. Translated by J. B. Brough.

Johansson, P., Hall, L., Sikström, S., \& Olsson, A. (2005). Failure to detect mismatches between intention and outcome in a simple decision task. Science, 310(5745), 116-119.

Kabat-Zinn, J. (1994). Wherever you go, there you are: Mindfulness meditation in everyday life. New York: Hyperion.

Kordeš, U. (2016). Going beyond theory: Constructivism and empirical phenomenology. Constructivist Foundations, 11(2), 202-223.

Kordeš, U., \& Demšar, E. (2018). Excavating belief about past experience: Experiential dynamics of the reflective act. Constructivist Foundations, 13(2), 219-229.

Kordeš, U., \& Demšar, E. (2019). Towards the epistemology of the non-trivial: Research characteristics connecting quantum mechanics and first-person inquiry. Foundations of Science. https://doi.org/10.1007/s10699-019-09638-z.
Kordeš, U., \& Markič, O. (2016). Parallels between mindfulness and first-person research into consciousness. Asian Studies, 4(2), 153-168.

Kordeš, U., Oblak, A., Smrdu, M., \& Demšar, E. (2019). Ethnography of meditation: An account of pursuing meditative practice as a tool for researching consciousness. Journal of Consciousness Studies, 26(7-8), 184-237.

Loftus, E. F., Miller, D. G., \& Burns, H. J. (1978). Semantic integration of verbal information into a visual memory. Journal of Experimental Psychology: Human Learning and Memory, 4(1), 19.

Metzinger, T. (2004). Being no one: The self-model theory of subjectivity. Cambridge MA: MIT Press.

Morley, J. (2001). Inspiration and expiration: Yoga practice through Merleau-Ponty's phenomenology of the body. Philosophy East and West, 73-82.

Morley, J. (2010). It's always about the epoché. Les Collectifs du Cirp, 1, 223-232.

Nichols, A. L., \& Maner, J. K. (2008). The good-subject effect: Investigating participant demand characteristics. The Journal of General Psychology, 135(2), 151-166.

Nisbett, R. E., \& Wilson, T. D. (1977). Telling more than we can know: Verbal reports on mental processes. Psychological Review, 84(3), 231.

Orne, M. T. (1962). On the social psychology of the psychological experiment: With particular reference to demand characteristics and their implications. American Psychologist, 17(11), 776-783.

Petitmengin, C. (2006). Describing one's subjective experience in the second person: An interview method for a science of consciousness. Phenomenology and the Cognitive Sciences, 5(3), 229-269.

Petitmengin, C. (2007). Towards the source of thoughts: The gestural and transmodal dimension of lived experience. Journal of Consciousness Studies, 14(3), 54-82.

Petitmengin, C. (2009). Editorial introduction. Journal of Consciousness Studies, 16 (10-12), 7-19.

Petitmengin, C., Bitbol, M., Nissou, J. M., Pachoud, B., Curalucci, C., Cermolacce, M., \& Vion-Dury, J. (2009). Listening from within. Journal of Consciousness Studies, 16 (10-12), 252-284.

Petitmengin, C., Remillieux, A., \& Valenzuela-Moguillansky, C. (2018). Discovering the structures of lived experience. Phenomenology and the Cognitive Sciences, 1-40.

Petitmengin, C., Van Beek, M., Bitbol, M., \& Nissou, J. M. (2017). What is it like to meditate? Methods and issues for a micro-phenomenological description of meditative experience. Journal of Consciousness Studies, 24(5-6), 170-198.

Ratcliffe, M. (2005). The feeling of being. Journal of Consciousness Studies, 12(8-9), 43-60.

Schooler, J., \& Schreiber, C. A. (2004). Experience, meta-consciousness, and the paradox of introspection. Journal of Consciousness Studies, 11(7-8), 17-39.

Searle, J. (1992). The rediscovery of the mind. Cambridge MA: MIT Press.

Sparby, T. (2015). Investigating the depths of consciousness through meditation. Mind and Matter, 13(2), 213-240.

Thompson, E. (2007). Mind in life: Biology, phenomenology, and the sciences of mind. Cambridge MA: Harvard University Press.

Thompson, E. (2014). Waking, dreaming, being: Self and consciousness in neuroscience, meditation, and philosophy. New York: Columbia University Press.

Varela, F. J. (1996). Neurophenomenology: A methodological remedy for the hard problem. Journal of Consciousness Studies, 3(4), 330-349.

Varela, F. J., \& Shear, J. (Eds.). (1999). View from within: First-person approaches to the study of consciousness. Exeter: Imprint Academic.

Varela, F. J., Thompson, E., \& Rosch, E. (1991). The embodied mind: Cognitive science and human experience. Cambridge MA: MIT Press.

Vermersch, P. (1994). L'entretien d'explicitation. Paris: ESF.

Wallace, B. A. (1999). The Buddhist tradition of Samatha: Methods for refining and examining consciousness. Journal of Consciousness Studies, 6(2-3), 175-187.

Zahavi, D. (2019). Applied phenomenology: Why it is safe to ignore the epoché. Continental Philosophy Review, 1-15. 